\title{
HSD3B2, HSD17B1, HSD17B2, ESR1, ESR2 and AR expression in infertile women with endometriosis
}

\author{
Maciej Osiński ${ }^{1}$, Przemysław Wirstlein ${ }^{1}$, Ewa Wender-Ożegowska' ${ }^{1}$, Mateusz Mikołajczyk ${ }^{1}$, \\ Paweł P. Jagodziński ${ }^{2}$, Małgorzata Szczepańska ${ }^{1}$ \\ ${ }^{1}$ Division of Reproduction, Department of Obstetrics, Gynaecology and Gynaecological Oncology, Poznan, Poland \\ ${ }^{2}$ Department of Biochemistry and Molecular Biology, Poznan University of Medical Sciences, Poznan, Poland
}

\begin{abstract}
Objectives: The development of endometriosis is associated with changes in the expression of genes encoding the $3 \beta$-hydroxysteroid dehydrogenase type II (HSD3B2) and 17 $\beta$-hydroxysteroid dehydrogenase type II (HSD17B2), estrogen receptors 1 (ESR1) and 2 (ESR2) and the androgen receptor (AR). However, little is known about the expression of HSD3B2, $H S D 17 B 1, H S D 17 B 2, E S R 1 E S R 2$ and $A R$ during the endometrial phases in eutopic endometrium from infertile women with endometriosis.
\end{abstract}

Material and methods: Using RT-qPCR analysis, we assessed the expression of the studied genes in the follicular and luteal phases in eutopic endometrium from fertile women $(n=17)$ and infertile women $(n=35)$ with endometriosis.

Results: In the mid-follicular eutopic endometrium, we observed a significant increase in HSD3B2 transcript levels in all infertile women with endometriosis $(p=0.003)$, in infertile women with stage $I / I$ endometriosis $(p=0.008)$ and in infertile women with stage III/IV endometriosis $(p=0.009)$ compared to all fertile women. There was a significant increase in ESR1 transcripts in all infertile women with endometriosis $(p=0.008)$ and in infertile women with stage $I / I$ endometriosis $(p=0.019)$ and in infertile women with stage III/IV endometriosis $(p=0.023)$ compared to all fertile women. In the mid-luteal eutopic endometrium, we did not observe significant differences in HSD3B2, HSD17B1, HSD17B2, ESR1, ESR2 and AR transcripts between infertile women with endometriosis and fertile women.

Conclusions: Observed significant increase in HSD3B2 and ESR1 transcripts in follicular eutopic endometrium from infertile women with endometriosis may be related to abnormal biological effect of E2 in endometrium, further affecting the development of human embryos.

Key words: fertility, endometriosis, genes expression

Ginekologia Polska 2018; 89, 3: 125-134

\section{INTRODUCTION}

Endometriosis is a disease characterized by the existence of functional ectopic endometrium outside the uterine cavity that affects $6-10 \%$ of women of reproductive age [1]. Three main etiopathogenic hypotheses regarding the development of endometriosis have been suggested [2,3]. The most widely accepted includes the retrograde shedding of endometriotic cells during menstruation inside the abdominal cavity, which results in a persistent inflammatory response [2,3]. Other theories explaining the development of these lesions include the coelomic metaplasia theory, which relates to the formation of endometrial tissue from the differentiation of mesothelium cells $[2,3]$. It has also been suggested that there is an embryonic origin of endometriosis [2, 3]. Depending on the severity of endometriosis, it can be subdivided into peritoneal, ovarian and deep lesions, which spread in the vagina, bowels, bladder or ureter $[4,5]$. Endometriosis is a complex disorder resulting from interactions between genetic and environmental factors $[6,7]$. The development of endometriosis is related to the abnormal expression of genes encoding proteins involved in vascular and tissue remodelling, enzymes involved in 
glucose homeostasis and proteins affecting sex hormone activity $[6,8]$. Endometriosis is also characterized by epigenetic disorders associated with the hypermethylation and hypomethylation of some gene promotors, modifications of the histone code and abnormal expression of various miRNAs involved in the pathogenesis of this disease [9].

However, the exact molecular mechanism accounting for the development of endometriosis and related infertility remains unclear [3]. To date, the expression of several genes involved in the metabolism and action of steroids have been demonstrated as being involved in endometriosis [10-13]. They include genes encoding $3 \beta$-hydroxysteroid dehydrogenase (HSD3B2) (OMIM * 613890), 17 $\beta$-hydroxysteroid dehydrogenase type I (HSD17B1) (OMIM * 109684), 17ß-hydroxysteroid dehydrogenase type II (HSD17B2) (OMIM* 109685), estrogen receptor 1 (ESR1) (OMIM +133430), estrogen receptor 2 (ESR2) (OMIM * 601663), and androgen receptor (AR) (OMIM * 313700). However, little is known about HSD3B2, $H S D 17 B 1, H S D 17 B 2, E S R 1, E S R 2$ and $A R$ expression in luteal and follicular eutopic endometrium in infertile women with endometriosis. Therefore, we assessed the expression of these genes during the follicular and luteal phases in eutopic endometrium from infertile women with endometriosis and fertile women.

\section{MATERIAL AND METHODS Patients and controls}

Data from fertile women and infertile women with endometriosis were randomly collected from the Gynaecologic and Obstetrical University Hospital, Division of Reproduction in Poznan, Poland (Tab. 1). The control group comprised fertile women who suffered from chronic pelvic pain, diagnosed as having varicose veins in the pelvic floor without any pelvic abnormalities and without a history of infertility.
The varicose veins in the pelvic floor were diagnosed by ultrasound method and confirmed via laparoscopy. The criteria for the sonographic diagnosis of varices included (1) the visualization of dilated ovarian veins greater than $4 \mathrm{~mm}$ in diameter, (2) dilated tortuous arcuate veins in the myometrium that communicate bilaterally with the pelvic varicose veins, (3) slow blood flow (less than 3 centimeters per second), and reversed caudal or retrograde venous blood flow particularly in the left ovarian vein [14].

The inclusion and exclusion criteria for the women without endometriosis and the infertile women with endometriosis were previously described [15]. During laparoscopy, we observed the condition of the pelvic cavity, uterus, fallopian tubes, ovaries, tubo-ovarian relationship, status of the Pounch of Douglas, and fimbrie. The inclusion criteria for the fertile control women were: regular menses, mobility of uterus, no anatomical changes in the reproductive tract, no hormonal treatments, and at least one child born no more than one year before the study (Tab. 1). The exclusion criteria were: signs of past or present inflammation. We didn't find inflamed fallopian tubes. In each patient, the peritoneal fluid was sampled for bacteriological examination, and was found to be sterile. Exclusion criteria also included pelvic abnormalities such as uterine fibroids, ovarian cysts, hydrosalpinges, adhesions in pouch of Douglas and the rest of the pelvis, endometriosis, adenomyosis, polycystic ovary syndrome (PCOS) or any other benign or malignant gynaecological diseases.

Patients with endometriosis were evaluated according to the revised American Society for Reproductive Medicine (rASRM) classification system [16] (Tab. 1). The inclusion criteria for infertile women with diagnosed endometriosis were: regular menses, no anatomical changes in the reproductive tract, no hormonal treatments and a minimum of one year of

Table 1. Clinical characteristics of women with endometriosis and controls

\begin{tabular}{|c|c|c|c|}
\hline \multicolumn{4}{|l|}{ Proliferative phase } \\
\hline Characteristic & I+ll stage of endometriosis ${ }^{b}$ & III+IV stage of endometriosis ${ }^{b}$ & Fertile Woman \\
\hline Numbers & 10 & 10 & 9 \\
\hline Age (years) & $36.5(25-39)^{\mathrm{a}}$ & $36(27-40)$ & $34(27-36)^{\mathrm{a}}$ \\
\hline Parity & NA & NA & $1(1-2)^{\mathrm{a}}$ \\
\hline Duration of infertility (years) & $2(1-4)^{a}$ & $2(2-3)^{a}$ & NA \\
\hline \multicolumn{4}{|l|}{ Luteal phase } \\
\hline Characteristic & I+ll stage of endometriosis ${ }^{b}$ & III+IV stage of endometriosis ${ }^{b}$ & Fertile Woman \\
\hline Numbers & 8 & 7 & 8 \\
\hline Age (years) & $35(27-38)^{a}$ & $35(25-41)$ & $34(28-40)^{\mathrm{a}}$ \\
\hline Parity & NA & NA & $1(1-3)^{\mathrm{a}}$ \\
\hline Duration of infertility (years) & $3(1-4)^{a}$ & $3(1-5)^{a}$ & NA \\
\hline
\end{tabular}

Median ${ }^{\mathrm{a}}$ (Range), revised American Society for Reproductive Medicine classification ${ }^{\mathrm{b}}$ (rASRM) [16]; NA — not applicable 
infertility with a current desire for conception. The exclusion criteria were: mechanical distortion of the endometrial cavity by fibroids, bilateral tubal occlusion, male factor infertility, adenomyosis, PCOS or benign or malignant gynaecological diseases. All included patients with endometriosis had laparoscopic and histological diagnosis of endometriotic lesions. The patients with endometriosis and the healthy controls were all Caucasian race of Polish ancestry (Tab. 1).

Samples were obtained by Pipelle or hysteroscopic biopsy of the eutopic endometrium during either the middle follicular or the middle luteal phase based on endometrial dating criteria [17]. Samples of mid-luteal eutopic endometrium tissue from patients and controls were collected during the implantation window, i.e., 7-9 days after ultrasound-confirmed ovulation. The eutopic endometrium samples were then used for total RNA and protein isolation.

\section{Reverse transcription and quantitative real-time PCR (RT-QPCR) analysis of HSD3B2, HSD17B1, HSD17B2, ESR1, ESR2 and AR transcript levels}

The obtained endometrial samples were placed overnight in Allprotect Tissue Reagent Solution (Qiagen $\mathrm{GmbH}$, Hilden, Germany) and frozen until extraction. Isolation of total RNA containing cytoplasmic mRNA was conducted with the use of the AllPrep DNA/RNA/Protein Mini Kit (Qiagen $\mathrm{GmbH}$, Hilden, Germany). For total RNA isolation we used, according to the manufacturer recommendation, an additional RNase-Free Dnase I Set (Qiagen $\mathrm{GmbH}$, Hilden, Germany) to eliminate the risk of DNA contamination. RNA quality was determined spectrophotometrically using a NanoDrop ND1000 (Thermo Fisher Scientific, Waltham, $\mathrm{MA}$ ) and agarose gel electrophoresis. RNA samples were reverse-transcribed (RT) into complementary DNA (cDNA) with the Quanti-Tect Reverse Transcription Kit (Qiagen GmbH, Hilden, Germany) (Tab. 2).

Quantitative analysis of HSD3B2, HSD17B1, HSD17B2, ESR1 ESR2 and AR CDNAs (Tab. 2) was performed by the Rotor-Gene 3000 thermocycler (Corbett Research, Australia), using SYBR Green I as the detection dye. HSD3B2, HSD17B1, HSD17B2, ESR1 ESR2 and AR CDNAs were quantified using the relative quantification method with a calibrator. The calibrator was prepared with a cDNA mix from all cDNA samples, and consecutive dilutions were used to create a standard curve. For amplification, $1 \mu$ l of the CDNA solution was added to $19 \mu$ l of the DyNAmo HS SYBR Green qPCR Kit (Thermo Fisher Scientific, Waltham, MA) and primers mix (Tab. 2).

Table 2. Detailed RT-qPCR procedure

\begin{tabular}{|c|c|}
\hline Definition of experimental and control groups & $\begin{array}{l}\text { Experimental group includes eutopic, proliferative and luteal endometrium from } \\
\text { infertile women with various stages of endometriosis defined according with revised } \\
\text { American Society for Reproductive Medicine (rASRM) classification system. Control } \\
\text { group includes eutopic, proliferative and luteal endometrium from fertile women } \\
\text { w/o endometriosis }\end{array}$ \\
\hline Number within each group & $\begin{array}{l}\text { Proliferative endometrium has } 12 \text { samples with minimal endometriosis (I, II st. rASRM), } \\
11 \text { samples with advanced endometriosis (III-IV st. RASRM) and } 9 \text { samples from fertile } \\
\text { women w/o endometriosis } \\
\text { Luteal endometrium has } 8 \text { samples with minimal endometriosis (I, II st. rASRM), } 7 \text { samples } \\
\text { with advanced endometriosis (III- IV st. RASRM) and } 9 \text { samples from fertile women } \\
\text { w/o endometriosis }\end{array}$ \\
\hline Acknowledgment of authors' contributions & Przemysław Wirstlein \\
\hline Description & Eutopic endometrium tissue \\
\hline Processing procedure & Immersed overnight in AllProtect Tissue Reagent (Qiagen, Germany) \\
\hline If frozen, how and how quickly? & Frozen $-20^{\circ} \mathrm{C}$ \\
\hline $\begin{array}{l}\text { Sample storage conditions and duration (especially } \\
\text { for } \mathrm{FFPE}^{\mathrm{b}} \text { samples) }\end{array}$ & $-20^{\circ} \mathrm{C}$ \\
\hline Procedure and/or instrumentation & Affinity, on columns \\
\hline Name of kit and details of any modifications & $\begin{array}{l}\text { AllPrep }{ }^{\circ} \text { DNA/RNA/Protein/MiniKit (50) ref. \# } 80004 \text {. In last step, protein was precipitated } \\
\text { with ice cold acetone, and resolved in 2\% SDS, according to Pierce TR0049.0 technical } \\
\text { resouce }\end{array}$ \\
\hline Source of additional reagents used & SDS obtained from Sigma Aldrich (Steinheim, Germany) \\
\hline Details of DNase or RNase treatment & RNA samples were treated with DNase I \\
\hline Contamination assessment (DNA or RNA) & qPCR amplification of genomic DNA fragment \\
\hline Nucleic acid quantification & spectrophotometrically \\
\hline Instrument and method & NanoDrop ND1000 (ThermoScientific, USA) \\
\hline RNA integrity: method/instrument & agarose gel electrophoresis \\
\hline
\end{tabular}


Table 2. (cont.) Detailed RT-qPCR procedure

Inhibition testing (Cq dilutions, spike, or other)

Complete reaction conditions

Amount of RNA and reaction volume

Priming oligonucleotide (if using GSP) and

concentration

Reverse transcriptase and concentration

Temperature and time

Manufacturer of reagents and catalogue numbers

Storage conditions of CDNA

Gene symbol

Sequence accession number (ENST)

Amplicon length

In silico specificity screen (BLAST, and so on)

Sequence alignment

Secondary structure analysis of amplicon

Location of each primer by exon or intron (if applicable)

What splice variants are targeted?

Primer sequences

Manufacturer of oligonucleotides
Cq dilutions of cDNA

1 ug of Total RNA was dissolved in 14ul final volume gDNA wipeout Buffer. After gDNA eliminaton step ( 2 min; $42^{\circ} \mathrm{C}$ ). Next, $4 \mu$ l Quantiscript RT Buffer (IncludesMg and dNTPs ), $1 \mu$ l Primer Mix (blended: oligo d(T) and hexamers) and $1 \mu \mathrm{l}$ Quantiscript Reverse Transcriptase (Contained Rnase inhibitor) was added to final volume $20 \mu \mathrm{l}$ ). Reverse transcription was performed $15 \mathrm{~min}$. at $42^{\circ} \mathrm{C}$ and $3 \mathrm{~min}$. at $95^{\circ} \mathrm{C}$

$1 \mu \mathrm{g}$ of RNA, reaction volume $20 \mu \mathrm{l}$

oligo $\mathrm{d}(\mathrm{T})$ and hexamers blended

The manufacturer didn't provaide detailed concentration

Quantiscript Reverse Transcriptase (Qiagen, Germany). The manufacturer didn't provaide details

according to the manufacturer's protocol

For RT Quantitect Reverse Transcription Kit, ref. \# 205311 (Qiagen, Germany)

$-20^{\circ} \mathrm{C}$

HSD3B2, HSD17B1, HSD17B2, ESR1, ESR2, AR,

00000543831 HSD3B2

00000585807 HSD17B1

00000199936 HSD17B2

00000440973 ESR1

00000554572 ESR2

00000374690 AR

127 HSD3B2

178 HSD17B1

181 HSD17B2

153 ESR1

166 ESR2

$138 \mathrm{AR}$

BLASTN 2.5.1+ (https://blast.ncbi.nlm.nih.gov/)

BLASTN 2.5.1+ (https://blast.ncbi.nlm.nih.gov/)

Oligo 7.6 software (http://www.oligo.net/downloads.html)

HSDB2: forward - exon 4, reverse - exon 4

HSD17B1: forward - exon 4, reverse - exon 5

HSD17B2: forward - exons junction 1/2, reverse - exon 2

ESR1: forward - exon 9, reverse - exon 9

ESR2: forward - exon 8, reverse - exon 9

AR: forward - exon 7, reverse - exon 7

HSD3B2-206

HSD17B1-202

HSD17B1-201

ESR1-207

ESR1-208

AR-201

HSD3B2 forward: 5'GCGGCTAATGGGTGGAATCTA 3'

HSD17B1 forward: 5'CGAAGGCTTATGCGAGAGTC 3'

HSD17B2 forward: 5'CTGGTGACAGGTGGTGATTG 3'

ESR1 forward: 5' AGCACCCTGAAGTCTCTGGA 3'

ESR2 forward: 5'TGGAGTCTGGTCGTGTGAAGA 3'

AR forward: 5' CAGTGGATGGGCTGAAAAAT 3'

HSD3B2 reverse: 5'CATTCTTGTTCAGGGCCTCAT 3'

HSD17B1 reverse: 5'GTGGGCGAGGTATTGGTAGA 3'

HSD17B2 reverse: 5'TTATCTGCACTGGCTTCGTG 3'

ESR1 forward: 5' GATGTGGGAGAGGATGAGGA 3'

ESR2 forward: 5' CTTCACCATTCCCACTTCGT 3

AR forward: 5' GGAGCTTGGTGAGCTGGTAG 3'

ACTB forward: 5'TCCTCCCTGGAGAAGAGCTCAC3'

ACTB reverse: 5'TGAAGGTAGTTTCGTGGATGC3'

RPLP0 forward: 5'GGCGACCTGGAAGTCCAACT3'

RPLP0 reverse: 5'CCATCAGCACCACAGCCTTC3'

TIB MOLBIOL Sp. z o.o.(Poznan, Poland; http://www.tib-molbiol.pl/) 
Table 2. (cont.) Detailed RT-qPCR procedure

\begin{tabular}{|c|c|}
\hline Purification method & Polyacrylamide gel electrophoresis \\
\hline Complete reaction conditions & $\begin{array}{l}10 \mu \mathrm{l} \text { Master Mix ( } 2 \times \text { conc., } 5 \mathrm{mM} \mathrm{MgCl} \text { incl. })++1 \mu \mathrm{F} \text { and R primer mix ( } 5 \mu \mathrm{M} \text { final conc. of } \\
\text { each one) }+1 \mu \mathrm{l} \mathrm{cDNA}+8 \mu \mathrm{l} \mathrm{H}_{2} \mathrm{O}\end{array}$ \\
\hline Reaction volume and amount of cDNA/DNA & Reaction volume $20 \mu \mathrm{l}(\mathrm{cDNA} 1 \mu \mathrm{l})$ \\
\hline Primer, (probe), $\mathrm{Mg}^{2+}$, and dNTP concentrations & Final concentrations: Primer $0.5 \mu \mathrm{M}, \mathrm{Mg}^{2+} 2.5 \mathrm{mM}$, dNTP $0.8 \mathrm{mM}$ \\
\hline Polymerase identity and concentration & Polymerase based on a hot start version of a modified Tbr DNA polymerase. \\
\hline Buffer/kit identity and manufacturer & DyNAmoTM HS SYBR ${ }^{\circledR}$ Green qPCR Kit Ref.\#F-410 (ThermoScientific, USA) \\
\hline Additives (SYBR Green I, DMSO, and so forth) & SYBR Green I \\
\hline Manufacturer of plates/tubes and catalog number & ThermoScientific 0,2 PCR Thermo-Tubes Ref.\#AB-0620 \\
\hline Complete thermocycling parameters & $\begin{array}{l}\text { Preincubation: } 15 \mathrm{~min} 95^{\circ} \mathrm{C}, \\
\text { Amplification: } 10 \mathrm{~s} 94^{\circ} \mathrm{C}, 10 \mathrm{~s} 55-60^{\circ} \mathrm{C}, 20 \mathrm{~s} 72^{\circ} \mathrm{C} \text { with single fluorescence acquisition, } \\
40 \text { cycles, Ramp rate: } 2.2^{\circ} \mathrm{C} \text { (cooling) and } 4.4^{\circ} \mathrm{C} \text { (heating) Melting: } 1 \mathrm{~min} 95^{\circ} \mathrm{C}, 1 \mathrm{~min} 40^{\circ} \mathrm{C} \text {, } \\
75-95^{\circ} \mathrm{C} \text { with continuous fluorescence acquisition }\end{array}$ \\
\hline Reaction setup (manual/robotic) & Manual \\
\hline Manufacturer of qPCR instrument & RotorGene3000 (Corbett Research, Australia) \\
\hline Evidence of optimization (from gradients) & PCR with gradient temperature of annealing and electrophoresis \\
\hline Specificity (gel, sequence, melt, or digest) & Melting curve \\
\hline For SYBR Green I, Cq of the NTC & NTC with no amplification observed $(\mathrm{Cq}>32)$ \\
\hline Calibration curves with slope and y intercept & $\begin{array}{l}\text { The PCR amplification efficiency for target and reference CDNA was determined by different } \\
\text { standard curves created by consecutive dilutions of the CDNA template mixture }\end{array}$ \\
\hline PCR efficiency calculated from slope & $\begin{array}{l}\text { HSD3B2 E }=1.000 \\
\text { HSD17B1-E }=0.999 \\
\text { HSD17B1-2E }=0.999 \\
E S R 1-E=1.000 \\
E S R 1-E=0.998 \\
A R-E=0.996\end{array}$ \\
\hline r2 of calibration curve & $R^{2}$ range from 0.9985 to 0.9997 \\
\hline Linear dynamic range & CDNA dilution from 1 to $1 / 10-4$ \\
\hline qPCR analysis program (source, version) & RotorGene 6. Version 6.1 (Build 93) (Corbett Research, Australia) \\
\hline Method of $\mathrm{Cq}$ determination & Second derivative maximum method \\
\hline Outlier identification and disposition & Pierce criterion \\
\hline Results for NTCs & No amplification observed for NTCs \\
\hline $\begin{array}{l}\text { Justification of number and choice of reference } \\
\text { genes }\end{array}$ & $\begin{array}{l}\text { Two reference genes: Actin, beta (ACTB) and Ribosomal Protein Lateral Stalk Subunit } \\
\text { PO (RPLPO) }\end{array}$ \\
\hline Description of normalization method & $\begin{array}{l}\text { Relative quantification method with a calibrator. The calibrator was prepared as a cDNA mix } \\
\text { from all cDNA samples and consecutive dilutions were used to create a standard curve. The } \\
\text { quantity of studied transcript in each sample was standardized by the geometric mean of } \\
\text { transcript levels }\end{array}$ \\
\hline $\begin{array}{l}\text { Number and stage (reverse transcription or qPCR) of } \\
\text { technical replicates }\end{array}$ & Three \\
\hline Statistical methods for results significance & the Kruskal-Wallis test with Dunn's post-hoc \\
\hline Software (source, version) & SigmaStat v.3.5 (Systat Software Inc; USA) \\
\hline
\end{tabular}

The HSD3B2, HSD17B1, HSD17B2, ESR1 ESR2 and AR transcript levels in each sample were standardized by the geometric means of the reference beta-actin (ACTB) and ribosomal protein stalk subunit PO (RPLP0) transcript levels. The PCR amplification efficiency for the target and reference CDNA was determined by different standard curves created by consecutive dilutions of the CDNA template mixture. The HSD3B2, HSD17B1, HSD17B2, ESR1 ESR2 and AR CDNA and ACTB and RPLPO CDNAs were amplified using the primer pairs presented in Table 2. The HSD3B2, HSD17B1, HSD17B2, ESR1 ESR2 and AR mRNA levels were expressed as multiples of these $C D N A$ concentrations in the calibrator. The $\mathrm{Y}$ axis presents relative quantity (RQ) of HSD3B2, HSD17B1, HSD17B2, ESR1 ESR2 and AR mRNA levels.

\section{Data analysis}

Statistical analyses were conducted using the Mann-Whitney rank sum test and the Kruskal-Wallis one-way 
ANOVA on ranks. Statistical analyses were conducted using Statistica version 10, 2011 (Stat Soft, Inc., Tulsa, USA).

\section{Ethics}

The study was conducted in accordance with the code of ethics of the Declaration of Helsinki and obtained the approval of the Local Ethical Committee of Poznan University of Medical Sciences (923/14/04.12.2014). Written informed consent was obtained from all participating individuals.

\section{RESULTS \\ Comparison of HSD3B2, HSD17B1, HSD17B2, ESR1, ESR2 and AR transcript levels in the mid-follicular eutopic endometrium between infertile women with endometriosis and fertile women}

The biopsy of eutopic endometrium was performed during the mid-follicular phase in 10 infertile women with endometriosis at $\mathrm{I} / \mathrm{II}$ severity stage and 10 infertile women with III/IV severity stage. The biopsy of eutopic endometrium was also performed in 9 healthy fertile women during the mid-follicular phase (Tab. 1).

In the mid-follicular eutopic endometrium, we observed a significant increase in HSD3B2 transcript levels in all infertile women with endometriosis $(p=0.003)$ and in patients with stage $I / I I$ endometriosis $(p=0.008)$ and stage III/IV endometriosis ( $p=0.009)$ compared to the levels in all fertile women (Fig. 1A). There were no significant differences in HSD17B1 transcript levels between all infertile women with endometriosis $(p=0.899)$, subgroups with stages $\mathrm{I} / \mathrm{II}$ endometriosis ( $p=0.222$ ) and stages III/IV endometriosis $(p=0.625)$ and fertile women (Fig. 1B). We found an increasing trend in HSD17B2 transcript levels, which was not statistically significant, in the mid-follicular eutopic endometrium in all infertile women with endometriosis $(p=0.071)$. We found a significantly increased HSD17B2 transcript level in the subgroup of patients with stages $\mathrm{I} / \mathrm{I}$ endometriosis $(p=0.029)$ but not in the stages III/IV subgroup $(p=0.351)$ (Fig. 1C). We observed a significant increase in ESR1 transcript levels in all infertile women with endometriosis $(p=0.008)$, those with stages $\mathrm{I} / \mathrm{II}$ endometriosis $(p=0.019)$ and those with stages III/IV endometriosis $(p=0.023)$ compared to the level in all fertile women (Fig. 1D). There was no significant increasing trend of ESR2 transcript levels in the mid-follicular eutopic endometrium in all infertile women $(p=0.079)$ with endometriosis, or in subgroups with stages $I / I I(p=0.056)$ and III/IV $(p=0.261)$ compared to the levels in fertile women (Fig. 1E). We did not find significant differences in AR transcript levels between all infertile women with endometriosis $(p=0.461)$ or subgroups with stages $\mathrm{I} / \mathrm{II}$ $(p=0.450)$ and III/IV ( $p=0.635)$ and fertile women (Fig. 1F).

\section{Comparison of HSD3B2, HSD17B1, HSD17B2, ESR1, ESR2 and AR transcript levels in the mid-luteal eutopic endometrium between infertile women with endometriosis and fertile women}

The biopsy of eutopic endometrium was performed during the mid-luteal phase in 8 infertile women with endometriosis at I/II severity stage and 7 with III/IV severity stage. The biopsy of eutopic endometrium was also was performed in 8 healthy fertile women during the mid-luteal phase (Tab. 1).

In the mid-luteal eutopic endometrium, we found a statistically insignificant increasing trend of HSD3B2 transcript levels in all infertile women with endometriosis ( $p=0.057)$ and in the subgroups with stages I/II $(p=0.055)$ but not in the subgroup with stages III/IV ( $p=0.225)$ when compared to the levels in fertile women (Fig. 2A). There were no significant differences between fertile women and all infertile women with endometriosis, the subgroup with stages I/II and the subgroup with stages III/IV for transcript levels of HSD17B1 ( $p=0.751, p=0.609, p=0.943$ respectively), HSD17B2 ( $p=0.812, p=0.798, p=0.937$, respectively), ESR1 ( $p=0.692, p=0.523, p=0.134$, respectively) and ESR2 ( $p=0.478, p=0.250, p=0.957$, respectively), or $A R$ ( $p=0.937, p=0.999, p=0.830$, respectively) (Fig. 2B-2F).

\section{DISCUSSION}

Endometriosis is an estrogen-influenced gynaecological disorder characterized by over-activity of the estrogen pathway, including increased production of 17-bestradiol (E2) and its action in the eutopic and ectopic endometrium.

HSD3B catalyses the oxidation and isomerization of d-5-3-beta-hydroxysteroid precursors into delta-4-ketosteroids resulting in the formation of all classes of steroids. The HSD3B also converts epiandrosterone, to 5a-androstan-3-one. HSD3B1 is expressed predominantly in the placenta and the skin, whereas HSD3B2 is expressed almost exclusively in the adrenal glands and the gonads [18]. To date there have been several studies that evaluated HSD3B2 expression in endometriosis [10-12]. Attar et al. (2009) demonstrated that HSD3B2 transcript levels are significantly upregulated in extraovarian (peritoneal) endometriotic tissues compared to eutopic endometrial tissue from endometriosis-free women [10].

It has also been found that interleukin-4 and prostaglandin E2 synergistically increased HSD3B2 transcript levels in endometrioma stromal cells [11]. Recently, Huhtinen et al. (2014) found significantly increased HSD3B2 transcript levels in extraovarian and ovarian endometriosis lesions compared to control eutopic endometrium in the luteal or follicular phase. In contrast, Huhtinen et al. (2011) have not 


\section{Mid-follicular endometrium}
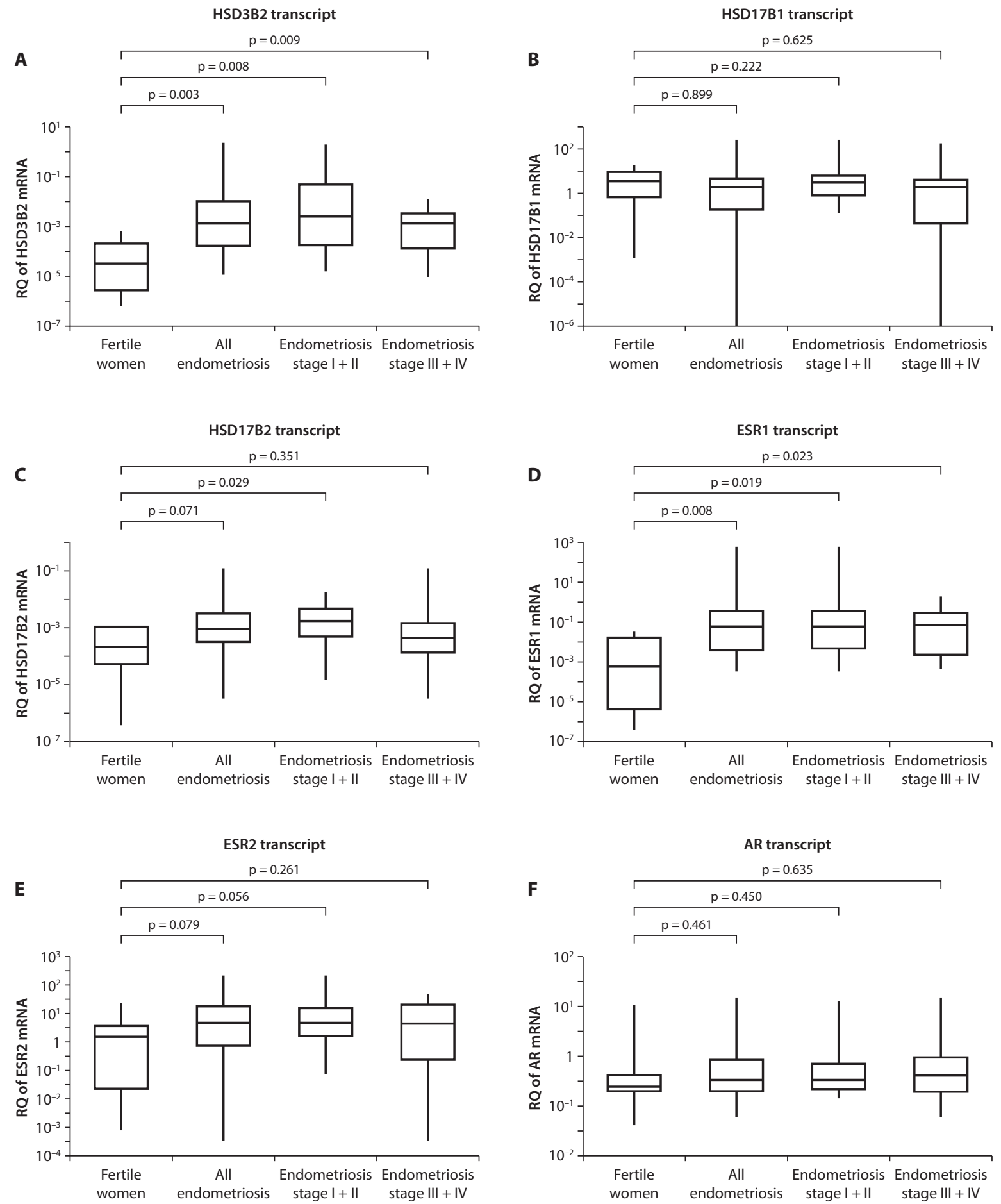

Figure 1. Comparison of HSD3B2 (A), HSD17B1 (B), HSD17B2 (C), ESR1 (D), ESR2 (E) and AR (F) transcript levels in the mid-follicular eutopic endometrium between fertile women and all infertile women with endometriosis, those with stages I/II endometriosis, and those with stages III/IV endometriosis.

Frozen tissue was homogenized, followed by total RNA isolation. Quantitative analyses of transcript levels were performed by RT-qPCR using the SYBR Green I system. The HSD3B2, HSD17B1, HSD17B2, ESR1, ESR2 and AR transcript levels in each sample were standardized by the geometric mean of references using ACTB and RPLPO cDNA levels. The P value was evaluated by the Mann-Whitney rank sum test and the Kruskal-Wallis test with pairwise multiple comparisons and post hoc Dunn's test. The boxes and the middle lines correspond to the values from the lower to upper quartiles and the medians, respectively. RQ-relative quantity 
Mid-luteal endometrium
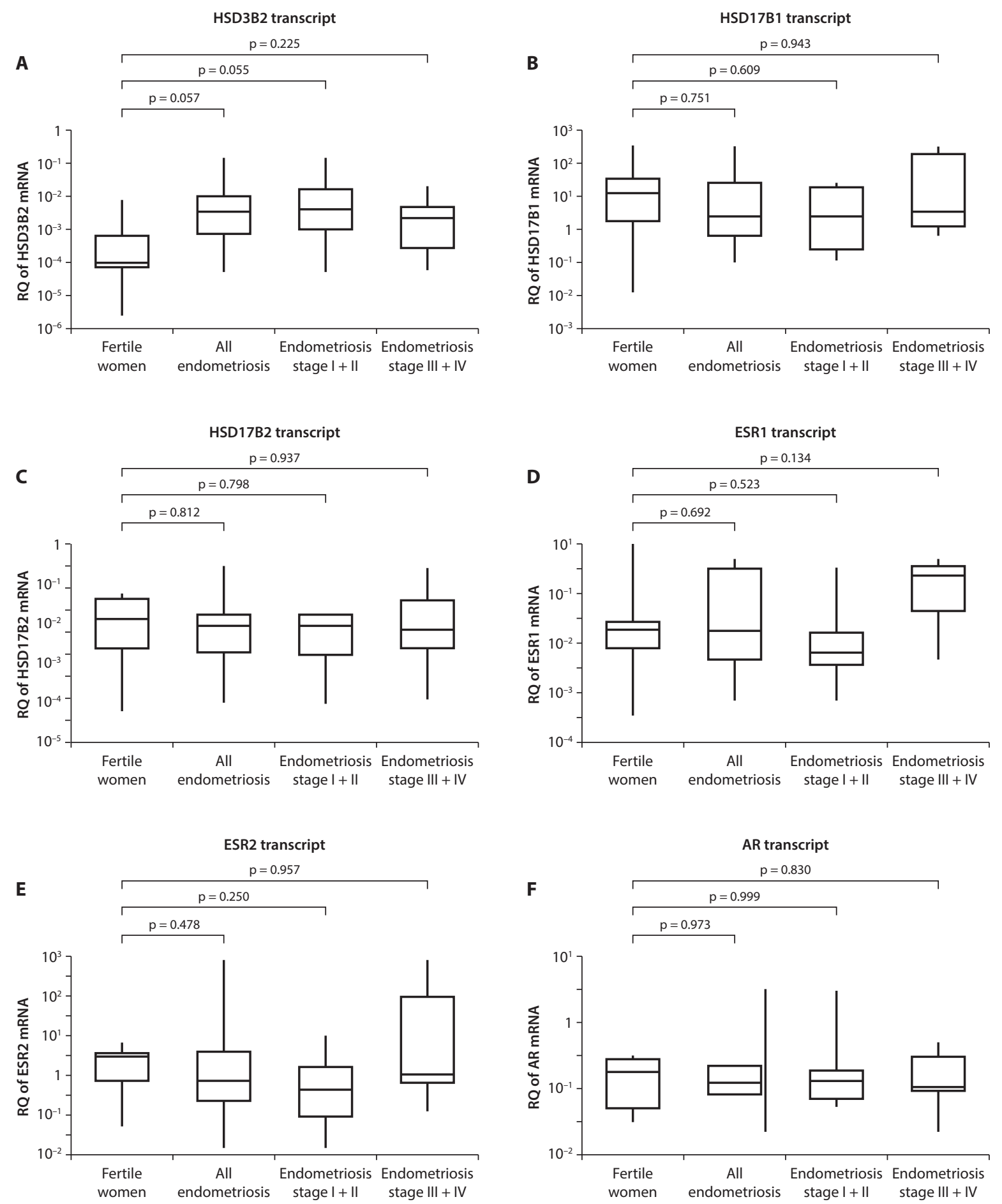

Figure 2. Comparison of HSD3B2 (A), HSD17B2 (B), HSD17B2 (C), ESR1 (D), ESR2 (E) and AR (F) transcript levels in the mid-luteal eutopic endometrium between fertile women and all infertile women with endometriosis, those with stages I/II endometriosis and those with stages III/IV endometriosis.

Frozen tissue was homogenized, followed by total RNA isolation. Quantitative analyses of transcript levels were performed by RT-qPCR using the SYBR Green I system. The HSD3B2, HSD17B1, HSD17B2, ESR1, ESR2 and AR transcript levels in each sample were standardized by the geometric mean of references using ACTB and RPLPO CDNA levels. The P value was evaluated by the Mann-Whitney rank sum test and the Kruskal-Wallis test with pairwise multiple comparisons and post hoc Dunn's test. The boxes and the middle lines correspond to the values from the lower to upper quartiles and the medians, respectively. RQ-relative quantity 
found any differences in HSD3B2 transcript levels between luteal and follicular phase eutopic endometrium from women with endometriosis and the eutopic endometrium of healthy women [12].

In our studies we found significantly increased levels of HSD3B2 transcript in the mid-follicular eutopic endometrium from all infertile women with endometriosis and in subgroups with stages I/II and III/IV when compared to the transcript levels in healthy women. In contrast, in the mid-luteal phase, there was no significant increasing trend of HSD3B2 transcripts in all infertile women with endometriosis or in the subgroup with stages $\mathrm{I} / \mathrm{II}$ versus the levels in the controls.

The presence of HSD17B1 and HSD17B2 oxidoreductases has been demonstrated in the eutopic endometrium [13]. HSD17B1 oxidizes estrone (E1) to E2, which is a more biologically active estrogen, while HSD17B2 reduces E2 to E1 [19]. Smuc et al. (2007) reported significantly increased HSD17B1 transcript levels in ectopic ovarian endometriosis compared to the levels in normal eutopic endometrium [13]. Delvoux_et al. (2013) demonstrated that inhibition of HSD17B1 can potentially be used as a treatment to restore the correct metabolism of estrogen in women with endometriosis with elevated local HSD17B1 activity [19]. However, in our study we did not find differences in HSD17B1 transcript levels between the eutopic endometrium of fertile women and that of infertile women with endometriosis.

Expression of HSD17B2 in the luteal phase is upregulated by progesterone in the endometrial glandular cells converting $E 2$ to $E 1$, the less biologically active estrogen [20]. Deficiency of the HSD17B2 enzyme impairs the inactivation of $E 2$ to $E 1$, which favours the accumulation of $E 2$ in endometriosis [21]. Zeitoun et al. (1998) demonstrated the presence of HSD17B2 transcripts and proteins in the luteal eutopic endometrium of healthy women but not in the luteal samples of endometriotic lesions [22]. Smuc et al. (2007) reported significantly decreased HSD17B2 transcript levels in ectopic ovarian endometriosis compared to normal endometrium [13] and HSD17B2 deficiency and locally increased E2 levels in ectopic implants in endometriosis [23]. In our studies, we found a statistically insignificant increasing trend of HSD17B2 transcript levels in the eutopic endometrium during the follicular phase but not in the luteal phase.

The estrogen receptor exists in two isoforms: ESR1 and ESR2 [24-25] with a 56\% homology between them [26]. ER activity depends on the binding of E2 and the nuclear receptor-induced transcription of ER-regulated genes. Research in the murine model has demonstrated that ESR1 plays the primary role in the uterus and neuroendocrine system, and female mice lacking ESR1 are infertile because of impaired ovarian and uterine function, while those lacking ESR2 display ovarian defects and subfertility [27]. The abnormal expression of ESR1 has been reported in endometriosis
$[13,28,29]$. Smuc et al. (2007) demonstrated that ESR1 transcript levels were downregulated in women with endometriosis group compared to the levels in the control group [13]. Using chromatin immunoprecipitation analysis, Monteiro et al. (2012) revealed the hypoacetylation of $\mathrm{H} 3 / \mathrm{H} 4$ histones inside the ESR1 promoter in endometriosis lesions but not in the control eutopic endometrium [28]. This hypoacetylation of $\mathrm{H} 3 / \mathrm{H} 4$ accounted for reduced ESR 1 expression in endometriosis lesions versus control eutopic endometrium [28]. Recently, Khan et al. (2017) suggested dysregulation of ESR1 expression as one of the significant causative factors in the pathogenesis of ovarian endometriosis [29] In our studies, we observed a significant increase in ESR1 transcript levels in the follicular but not the luteal phase in all infertile women with endometriosis and in the subgroups with stages I/II and stages III/IV.

The knowledge of biologic roles of ESR2 in the endometrium and in endometriosis is still elusive [30]. Smuc et al. (2007) demonstrated that ESR2 transcript levels were upregulated in the endometriosis group versus the control group [13]. An ESR2-selective compound has been demonstrated to have therapeutic activity in a rodent endometriosis model [30]. Our study only demonstrated an insignificant increase in ESR2 transcript levels in the mid-follicular eutopic endometrium in all infertile women with endometriosis and in the subgroup with stages $\mathrm{I} / \mathrm{II}$.

To date, $A R$ gene CAG repeat polymorphisms have been associated with an increased risk for mild endometriosis [31]. However, our results did not reveal any significant changes in the expression of $A R$ in the proliferative and luteal phases between infertile women with endometriosis and fertile women.

The differences between our results and others in terms of expression might be due to the employment of ectopic implants, despite the eutopic endometrium of endometriosis. Studies usually compare the expression of genes encoding proteins involved in estrogen activity in ectopic implants of endometriosis independent of the menstrual cycle phases [10-13, 22, 23, 28, 29]. We conducted our study using primary eutopic endometrium consisting of a mix of uterine gland, columnar epithelium and stromal fibroblast cells. Our study was also conducted in selected infertile women with endometriosis with determined menstrual phase cycles. However, our study has limitations with the very small number of studied cases and needs further evaluation of proteins using either immunohistochemistry or western blot.

\section{CONCLUSIONS}

Observed significant increase in HSD3B2 and ESR1 transcripts in follicular eutopic endometrium from infertile women with endometriosis may be related to abnormal 
biological effect of $E 2$ in endometrium, further affecting the development of human embryos.

\section{ACKNOWLEDGEMENTS}

The study has been supported by grant No 502-0101124182-07474 from the Poznań University of Medical Sciences, Poland.

\section{REFERENCES}

1. Luca A, Nemescu D, Butnaru M, et al. Ovarian stimulation outcome in infertile women with endometriosis undergoing IVF. Ginekol Pol. 2016; 87(1): 37-41, doi: 10.17772/gp/60073, indexed in Pubmed: 27306467.

2. Zubrzycka A, Zubrzycki M, Janecka A, et al. New Horizons in the Etiopathogenesis and Non-Invasive Diagnosis of Endometriosis. Curr Mol Med. 2015; 15(8): 697-713, indexed in Pubmed: 26391550.

3. Vercellini $P$, Viganò $P$, Somigliana $E$, et al. Endometriosis: pathogenesis and treatment. Nat Rev Endocrinol. 2014; 10(5): 261-275, doi: 10.1038/nrendo.2013.255, indexed in Pubmed: 24366116.

4. Wilczyński M, Wiecka-Płusa M, Antosiak B, et al. [Rectovaginal endometriosis--analysis of 160 cases]. Ginekol Pol. 2015; 86(12): 896-901, indexed in Pubmed: 26995938.

5. Ianieri MM, Mautone D, Ceccaroni M. Recurrence in Deep Infiltrating Endometriosis: A Systematic Review of the Literature. J Minim Invasive Gynecol. 2018 [Epub ahead of print], doi: 10.1016/j.jmig.2017.12.025, indexed in Pubmed: 29357317.

6. Krishnamoorthy K, Decherney AH. Genetics of Endometriosis. Clin Obstet Gynecol. 2017; 60(3): 531-538, doi: 10.1097/GRF.0000000000000293, indexed in Pubmed: 28742585.

7. Jurkiewicz-Przondziono J, Lemm M, Kwiatkowska-Pamuła A, et al. Influence of diet on the risk of developing endometriosis. Ginekol Pol. 2017; 88(2): 96-102, doi: 10.5603/GP.a2017.0017, indexed in Pubmed: 28326519.

8. Tanbo T, Fedorcsak P. Endometriosis-associated infertility: aspects of pathophysiological mechanisms and treatment options. Acta Obstet Gynecol Scand. 2017; 96(6): 659-667, doi: 10.1111/aogs.13082, indexed in Pubmed: 27998009.

9. Borghese B, Zondervan KT, Abrao MS, et al. Recent insights on the genetics and epigenetics of endometriosis. Clin Genet. 2017; 91(2): 254-264, doi: $10.1111 /$ cge.12897, indexed in Pubmed: 27753067.

10. Attar $\mathrm{E}$, Tokunaga $\mathrm{H}$, Imir $\mathrm{G}$, et al. Prostaglandin E2 via steroidogenic factor-1 coordinately regulates transcription of steroidogenic genes necessary for estrogen synthesis in endometriosis. J Clin Endocrinol Metab. 2009; 94(2): 623-631, doi: 10.1210/jc.2008-1180, indexed in Pubmed: 19001523.

11. Urata $Y$, Osuga $Y$, Akiyama I, et al. Interleukin-4 and prostaglandin E2 synergistically up-regulate $3 \beta$-hydroxysteroid dehydrogenase type 2 in endometrioma stromal cells. J Clin Endocrinol Metab. 2013; 98(4): 1583-1590, doi: 10.1210/jc.2012-3475, indexed in Pubmed: 23450050.

12. Huhtinen $K$, Saloniemi-Heinonen $T$, Keski-Rahkonen $P$, et al. Intra-tissue steroid profiling indicates differential progesterone and testosterone metabolism in the endometrium and endometriosis lesions. J Clin Endocrinol Metab. 2014; 99(11): E2188-E2197, doi: 10.1210/jc.2014-1913, indexed in Pubmed: 25137424.

13. Smuc T, Pucelj MR, Sinkovec J, et al. Expression analysis of the genes involved in estradiol and progesterone action in human ovarian endometriosis. Gynecol Endocrinol. 2007; 23(2): 105-111, doi: 10.1080/09513590601152219, indexed in Pubmed: 17454161.

14. Coakley FV, Varghese SL, Hricak H. CT and MRI of pelvic varices in women. J Comput Assist Tomogr. 1999; 23(3): 429-434, indexed in Pubmed: 10348450.
15. Szczepańska M, Wirstlein P, Skrzypczak J, et al. Polymorphic variants of CYP17 and CYP19A and risk of infertility in endometriosis. Acta Obstet Gynecol Scand. 2013; 92(10): 1188-1193, doi: 10.1111/aogs.12210, indexed in Pubmed: 23809139.

16. Hoeger K, Guzick D. CLASSIFICATION OF ENDOMETRIOSIS. Obstetrics and Gynecology Clinics of North America. 1997; 24(2): 347-359, doi: 10.1016/s0889-8545(05)70308-9.

17. Noyes RW, Hertig AT, Rock J. Dating the endometrial biopsy. Am J Obstet Gynecol. 1975; 122(2): 262-263, indexed in Pubmed: 1155504.

18. Goswami AM. Structural modeling and in silico analysis of non-synon-

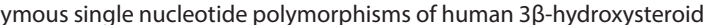
dehydrogenase type 2. Meta Gene. 2015; 5: 162-172, doi: 10.1016/j. mgene.2015.07.007, indexed in Pubmed: 26288759.

19. Delvoux B, D'Hooghe T, Kyama $C$, et al. Inhibition of type 1 $17 \beta$-hydroxysteroid dehydrogenase impairs the synthesis of $17 \beta$-estradiol in endometriosis lesions. J Clin Endocrinol Metab. 2014; 99(1): 276-284, doi: 10.1210/jc.2013-2851, indexed in Pubmed: 24187399.

20. Young SL. Oestrogen and progesterone action on endometrium: a translational approach to understanding endometrial receptivity. Reprod Biomed Online. 2013; 27(5): 497-505, doi: 10.1016/j.rbmo.2013.06.010, indexed in Pubmed: 23933037.

21. Sanchez AM, Somigliana E, Vercellini $P$, et al. Endometriosis as a detrimental condition for granulosa cell steroidogenesis and development: From molecular alterations to clinical impact. J Steroid Biochem Mol Biol. 2016; 155(Pt A): 35-46, doi: 10.1016/j.jsbmb.2015.07.023, indexed in Pubmed: 26407755.

22. Zeitoun K, Takayama K, Sasano H, et al. Deficient 17beta-hydroxysteroid dehydrogenase type 2 expression in endometriosis: failure to metabolize 17beta-estradiol. J Clin Endocrinol Metab. 1998; 83(12): 4474-4480, doi: 10.1210/jcem.83.12.5301, indexed in Pubmed: 9851796.

23. Bulun SE, Monsivais D, Kakinuma T, et al. Molecular biology of endometriosis: from aromatase to genomic abnormalities. Semin Reprod Med. 2015; 33(3): 220-224, doi: 10.1055/s-0035-1554053, indexed in Pubmed: 26036904

24. Sukocheva OA. Estrogen, estrogen receptors, and hepatocellular carcinoma: Are we there yet? World J Gastroenterol. 2018; 24(1): 1-4, doi: 10.3748/wjg.v24.i1.1, indexed in Pubmed: 29358876.

25. Catanuto $P, X i a X$, Pereira-Simon $S$, et al. Estrogen receptor subtype ratio change protects against podocyte damage. Curr Trends Endocinol.2017; 9: 19-29, indexed in Pubmed: 29367812.

26. Powles TJ. Anti-oestrogenic prevention of breast cancer--the make or break point. Nat Rev Cancer. 2002; 2(10): 787-794, doi: 10.1038/nrc908, indexed in Pubmed: 12360281.

27. Hamilton KJ, Hewitt SC, Arao Y, et al. Estrogen Hormone Biology. Curr Top Dev Biol. 2017; 125: 109-146, doi: 10.1016/bs.ctdb.2016.12.005, indexed in Pubmed: 28527569.

28. Monteiro JB, Colón-Díaz M, García M, et al. Endometriosis is characterized by a distinct pattern of histone 3 and histone 4 lysine modifications. Reprod Sci. 2014; 21(3):305-318, doi: 10.1177/1933719113497267, indexed in Pubmed: 23899551.

29. Khan MA, Sengupta J, Mittal S, et al. Genome-wide expressions in autologous eutopic and ectopic endometrium of fertile women with endometriosis. Reprod Biol Endocrinol. 2012; 10: 84, doi: 10.1186/14777827-10-84, indexed in Pubmed: 23006437.

30. Harris HA, Bruner-Tran KL, Zhang X, et al. A selective estrogen receptor-beta agonist causes lesion regression in an experimentally induced model of endometriosis. Hum Reprod. 2005; 20(4): 936-941, doi: 10.1093/humrep/deh711, indexed in Pubmed: 15618247.

31. Shin JJ, Choi YM, Choi HY, et al. Androgen receptor cytosine, adenine, and guanine trinucleotide repeat polymorphism in Korean patients with endometriosis: A case-control study. Eur J Obstet Gynecol Reprod Biol. 2017; 218: 1-4, doi: 10.1016/j.ejogrb.2017.09.005, indexed in Pubmed: 28915409. 